\title{
Sub-Millimeter Crack Detection in Casted Steel using Color Photometric Stereo
}

\author{
Anders Landström, Matthew J. Thurley, and Håkan Jonsson \\ Department of Computer Science, Electrical and Space Engineering \\ Luleå University of Technology \\ Luleå, Sweden \\ \{anders.p.landstrom, matthew.thurley, hakan.jonsson\}@ltu.se
}

\begin{abstract}
A novel method for automated inspection of small corner cracks in casted steel is presented, using a photometric stereo setup consisting of two light sources of different colors in conjunction with a line-scan camera. The resulting image is separated into two different reflection patterns which are used to cancel shadow effects and estimate the surface gradient. Statistical methods are used to first segment the image and then provide an estimated crack probability for each segmented region.

Results show that true cracks are successfully assigned a high crack probability, while only a minor proportion of other regions cause similar probability values. About $80 \%$ of the cracks present in the segmented regions are given a crack probability higher than $70 \%$, while the corresponding number for other non-crack regions is only $5 \%$. The segmented regions contain over $70 \%$ of the manually identified crack pixels. We thereby provide proofof-concept for the presented method.
\end{abstract}

Index Terms-Surface inspection, Crack detection, Photometric stereo.

\section{INTRODUCTION}

\section{A. Background}

Cracks in casted steel constitute a quality issue for enduser products and may also cause problems in subsequent production processes such as sheet steel rolling. Finding cracks after casting, thereby avoiding related problems at later stages, is therefore important to the steel industry. Much of this inspection is still manually operated, while a robust automated system can improve both working conditions and production efficiency. Moreover, automated methods provide an objective and consistent result which can be more easily analyzed than manual inspection. Moreover, in order to enable on-line testing of produced goods inspection must be done by NonDestructive Testing (NDT).

There exists many different NDT methods for defect detection in steel, such as thermocouples [1], eddy currents [2], ultra sound [3], conoscopic holography [4], and laser triangulation [5]. In this work however, we focus on the detection of small corner cracks which are in general thinner than $1 \mathrm{~mm}$. Due to their size such cracks pose a challenge to the techniques listed above when an on-line installation is desired.

Light intensity imaging on the other hand, i.e. standard gray-scale camera technique, enables fast acquisition of high resolution surface data suitable for on-line NDT installations, and has been used throughout the years for various inspection tasks [6]. Gray-scale images are often hard to interpret though, motivating the use of more advanced techniques. We present a photometric stereo system for identifying small cracks with sub-millimeter width in casted steel slabs using machine vision, enabling appropriate processing of such regions before slabs are shipped further down the production chain.

In photometric stereo, originally introduced by Woodham [7], multiple gray-scale images are acquired of the same scene under illumination by differently placed light sources. Assuming a surface is Lambertian (i.e. scatters light isotropically), its shape can be uniquely determined from three images obtained by illuminating the scene from three different directions. The different illumination patterns are then translated into height gradient information for the surface by solving a linear system of equations. Two-source photometric stereo gives a less well defined surface, but conditions for existence and uniqueness of the system of equations for the two-source case have been investigated by Kozera [8].

Most methods based on photometric stereo relies on data captured by taking several photos of the same scene, varying the origin of illumination using a set of light sources lit one at a time, but this approach would be cumbersome for the intended use. Instead, it should be possible to mount the equipment in an

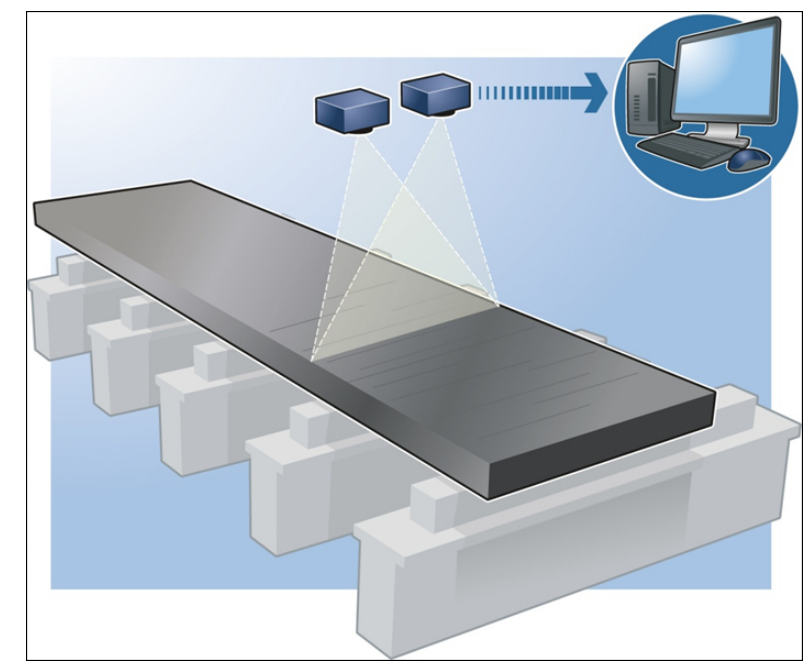

Fig. 1. The intended use of the final system: Scanning slabs from a measurement frame under which slabs can be placed for inspection. 
on-line measurement rig passing over the steel slabs, scanning the steel below (see Fig. 1). In order to enable such on-line scanning the images should be acquired simultaneously, which can be achieved by color photometric stereo.

Drew [9] estimates surface gradients from the different channels in a color image, using photometric stereo. No particular light setup is addressed, but conditions are imposed on the light setup by the underlying mathematical expressions, i.e. light directions must be linearly independent and colors must not be coplanar in RGB space. Color images have also been used by Balschbach et al. [10], who obtain height gradients for highly specular moving (wavy) water surfaces from refracted colored lights.

Kang et al. [11] recently used colored light photometric stereo to retrieve signatures for artificial defects in planar steel surfaces. Their work considers surface reconstruction from gradients obtained from a configuration based on three and six differently colored light sources. However, surface reconstruction from gradients is quite susceptible to noise in the data and therefore requires very accurate input when addressing thin defects such as cracks.

\section{B. Contribution}

The presented approach captures an estimated surface gradient from the different light reflections of two colored light sources. In addition, the use of two light sources also allows for separation between cracks and various shadowing effects. Working directly with the gradient makes the system more robust to noise, as compared to integrating gradients obtained by photometric stereo into a surface. Moreover, combining estimated gradient light intensities with gradient information provides a more robust identification than purely intensitybased approaches since the texture of the steel surface may affect the intensity of the reflected light. The work can be separated into three interrelated subproblems:

$P 1$. Acquiring image data that captures the details of small surface cracks.

$P 2$. Segmenting the image into possible crack regions.

$P 3$. Classifying potential cracks as true or false signatures.

The rest of this article is arranged as follows: Section II presents solutions to the three subproblems P1-P3, based on a model set of steel samples containing cracks. Results for the presented method on another set of samples is then presented in Section III, followed by an analysis in Section IV and conclusions in Section V. Future challenges and possibilities for the ongoing work are then addressed in Section VI.

\section{METHOD}

\section{A. Measurement Setup (PI)}

A transversal crack is characterized by a rapid change in the height derivative in the perpendicular direction, and will remain dark (low light intensity) independent of whether the projected light comes from the front or back. A solution for subproblem $P l$ must provide a high enough resolution to be able to get a clear signature of small cracks, but should be robust against variation of texture and reflections in the steel surface.

Photometric stereo enables simple and cost-efficient estimation of the surface gradient (i.e. slope). In addition, the two light sources enables us to retrieve a gray-scale intensity image of the sample where shadow effects appearing in only one of the images can be cancelled. We present a system which uses constant light of two different colors (yellow and blue) which can be well separated in the green and blue channels of the digital image captured by the camera.

The gradient, or 1:st derivative, is measured perpendicular to the cracks using two light sources $L_{1}$ and $L_{2}$ that are positioned in front of and behind the camera sensor, with respect to the relative movement between the camera and the slab. Thus, the gradient estimation is done longitudinally along the measured slab: the direction for which $L_{1}$ and $L_{2}$ are colinear (see Fig. 2). A line scan color camera is used to capture one transversal line of data at the time, located at constant and equal distance relative to $L_{1}$ and $L_{2}$. This setup avoids the need for position-dependent normalization of intensity values, simplifying mathematical expressions considerably.

Assuming a Lambertian surface (i.e. isotropic light scattering), a coarse but simple estimation of the surface gradient can be achieved by considering how light incident from different directions is reflected (i.e. in what extent it reaches the sensor). Given the symmetrical co-linear measurement setup, the system of equations for two-light photometric stereo considered by Kozera [8], among others, reduces to a normalized difference of the two images for the longitudinal direction (note that nothing is known about the transversal direction). That is, an approximation of the gradient $S_{y}$ of the surface $S$ in the longitudinal $(y-)$ direction of the slab can be obtained from the expression

$$
S_{y}=\frac{I_{1}-I_{2}}{I_{1}+I_{2}}
$$

where $I_{1}$ and $I_{2}$ are images captured while illuminating the surface from (one of) $L_{1}$ and $L_{2}$, respectively. For simplicity, we here omit a factor yielded by the angle of the incident light since it only scales the quantity. In a slightly different

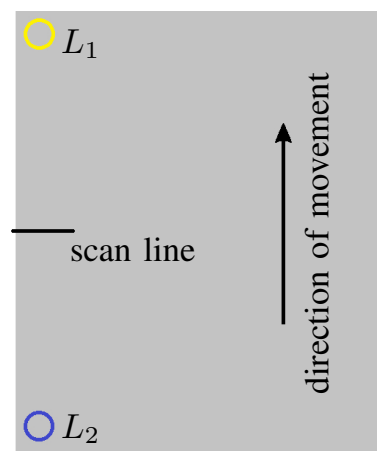

(a)

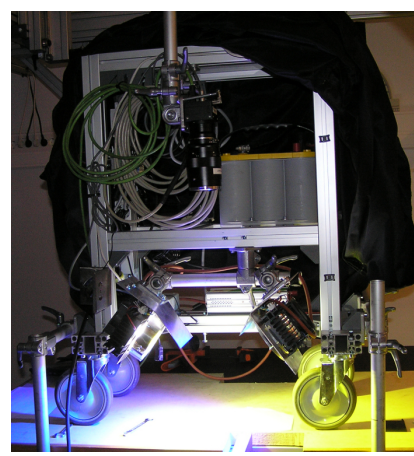

(b)
Fig. 2. (a) Setup example with light sources in blue and yellow, and scan line in black. The gray area represents a part of the slab. (b) The portable measurement setup installation used in this work. 
but related application, Nayar and Bolle [12] demonstrated how the same ratio of image intensities can be used to obtain a reflectance ratio for neighboring points in the same grayscale image, assuming identical light scattering for the two points.

It should be noted that a steel surface hardly provides perfect conditions, and consequentially the resulting estimated gradient should not be expected to yield an exact slope due to the risk of specular effects and similar disturbances. Steel is also hardly not a Lambertian surface, and a more accurate model for light scattering should yield a better gradient estimation. Nevertheless, empirical studies show that the simple approximation provided by (1) produces a highly usable result.

\section{B. Segmentation $(P 2)$}

We identify three key criteria for pixels within captured cracks:

C1. Low light intensity.

$C 2$. Low light intensity in relation to the surrounding region (not necessarily the same as the above criterion).

C3. Rapid (positive) change in the perpendicular height derivative.

Rather than defining thresholds for the above criteria manually we then apply a probabilistic approach, combining them into a pixelwise crack probability value based on a logistic regression model constructed from a model set of images containing in total over 50 cracks of different sizes.

An example of a pair of input images $I_{1}$ and $I_{2}$ are shown in Fig. 3. We assume the placement of the steel is well known, which means we can safely restrict filtering and classification to the center part of the acquired data. This decreases computation time and avoids false detection of cracks in non-interesting regions. A standard centimeternumbered ruler is displayed on the left side of the images. The difference in scale between the top and bottom of the images is a result of variation of the speed at which the line scan camera was swept over the sample. This demonstrates how different resolutions in the longitudinal $(\mathrm{y}-)$ direction can

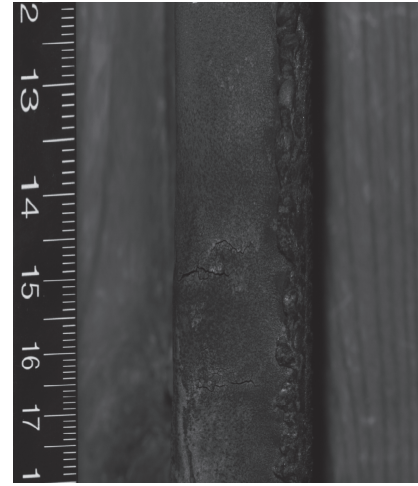

(a)

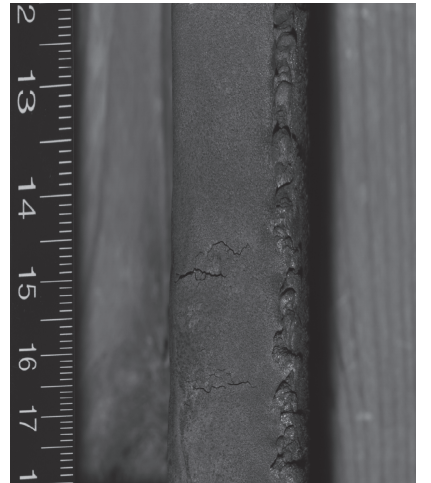

(b)
Fig. 3. The front and back images ( $a$ and b, respectively) for a steel sample in the model set, where cracks are visibile on the left side of the scarfed steel surface. The scale numbers to the left are denoted in centimeters. be obtained by varying the speed of the camera vs. the scanned slab.

1) Criterion C1: To cancel shadowing effects, we define the maximum intensity image $C_{1}$ as the pixelwise maximum of $I_{1}$ and $I_{2}$, filtered in order to enhance elongated dark features, i.e.

$$
C_{1}=\phi_{E}\left(\max \left\{I_{1}, I_{2}\right\}\right) .
$$

Here, $\phi_{E}$ denotes an adaptive morphological opening using elliptical structuring elements. This method was introduced in Ref. [13] as a method for enhancing and linking elongated features such as cracks. Maximum length of the semi-major axes of the elliptical structuring elements used to probe the image is set to 3 pixels.

2) Criterion $C 2$ : In the image $C_{1}$, shadows that are not present in both images are cancelled while regions appearing dark in both images, such as cracks, remain. Furthermore, we also capture the pixel darkness in relation to the surrounding neighborhood by calculating the morphological close tophat $C_{2}$, i.e. the difference between the morphological (nonadaptive) closing $\psi\left(C_{1}\right)$, using a reasonably large disk structuring element (radius 15 pixels), and $C_{1}$ itself:

$$
C_{2}=\psi\left(C_{1}\right)-C_{1} \text {. }
$$

Examples of the resulting images $C_{1}$ and $C_{2}$ are presented in Figs. $4 \mathrm{a}$ and $4 \mathrm{~b}$.

3) Criterion C3: Ideally the height derivative (slope) of the surface should turn from negative to positive, but this is not always true since the estimated derivative is only an approximation. For our third criterion we therefore simply consider the value of the $2^{\text {nd }}$ derivative (which should be high where there are cracks), as estimated from the photometric stereo setup. The 1:st derivative $S_{y}$ of the surface $S$ is estimated by (1), i.e.

$$
S_{y}=\frac{I_{1}-I_{2}}{I_{1}+I_{2}}
$$

and a measure $C_{3}$ related to our third criterion is then obtained from an estimation of the $2^{\text {nd }}$ derivative. This is done by first convolving the image $S_{y}$ with a small 2D Gaussian derivative kernel $D_{G}$ (the standard deviation used for the Gaussian is as small as 0.5 pixels in order to capture the thin details of the crack) and then linking signatures with adaptive morphological filtering in the same way as for $C_{1}$, i.e.

$$
C_{3}=\psi_{E}\left(S_{y} * D_{G}\right) .
$$

Here a closing $\psi_{E}$ is performed instead of an opening in order to enhance bright, rather than dark, features in the data. The maximum semi-major axis of the structuring elements is set to 3 pixels here as well. An example of a resulting image is shown in Fig. 4c.

4) Probabilistic Segmentation: As discussed (see the beginning of Section II-B), we apply a statistical method to separate between pixels which are likely or unlikely to constitute a part of a crack: a logistic regression model is fitted to the model data set, providing a posterior crack probability for each pixel 


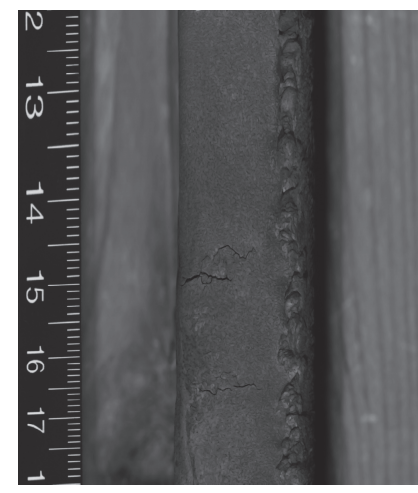

(a)

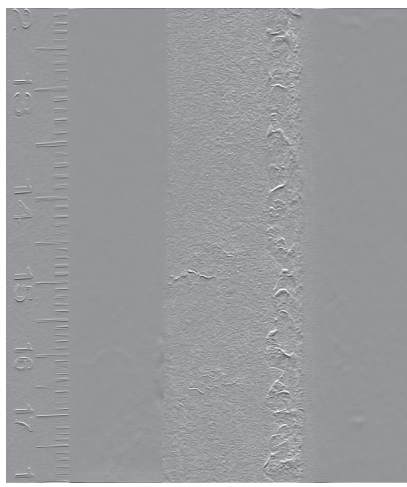

(c)

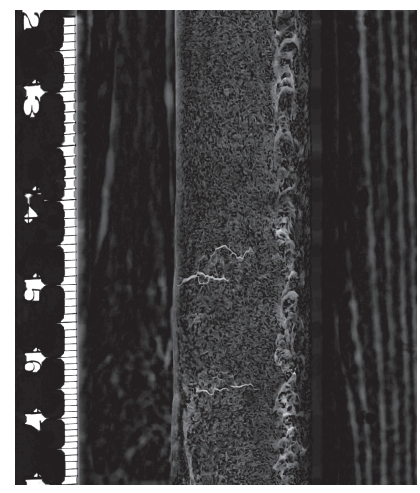

(b)

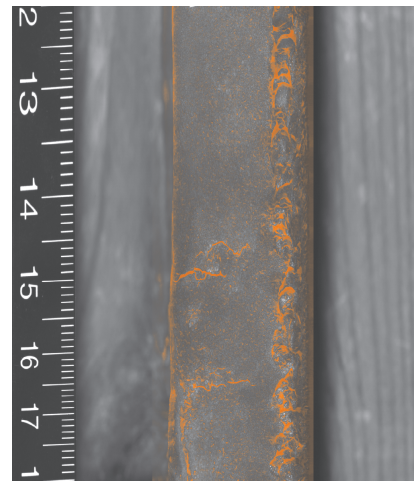

(d)
Fig. 4. The maximum intensity $C_{1}$ (a), its top-hat $C_{2}$ (b), the $2^{\text {nd }}$ derivative $S_{y y}$ (c), and the resulting estimated per-pixel crack probability shaded in orange (d). The brightness of the orange shading is proportional to the assigned crack probability, i.e. regions with high crack probability are shaded in bright orange.

based on the criteria of manually marked cracks in the model set (see Fig. 4d). More specifically, given a criteria vector $\mathbf{C}(\mathbf{x})=\left\{C_{1}(\mathbf{x}), C_{2}(\mathbf{x}), C_{3}(\mathbf{x})\right\}$ for a pixel $\mathbf{x}$ we define a corresponding crack probability $p(\mathbf{x} \mid \alpha, \mathbf{C})$ by the posterior probability for logistic regression

$$
p(\mathbf{x} \mid \alpha, \mathbf{C})=\frac{1}{1+e^{-\alpha^{T} \mathbf{C}(\mathbf{x})}},
$$

where $\alpha$ denotes the coefficients vector for the classifier obtained from the model set. A comparison of the value distributions for the variables and the pixel-wise crack probability (for the model set) is shown in Fig. 5.

The data is then segmented into a thresholded binary image $T$ by selecting a probability threshold $t$, i.e.

$$
T= \begin{cases}1, & \text { where } p(\mathbf{x} \mid \alpha, \mathbf{C}) \geq t \\ 0, & \text { otherwise }\end{cases}
$$

Based on the cumulative distribution functions (Fig. 5d) we select $t=0.75$, keeping pixels with more than $75 \%$ crack probability (as defined by the model set). This yields a set of segmented regions (see Fig. 6). A binary segmentation has thus been achieved by selecting one probability threshold rather than three variable thresholds. It should be noted that classification by logistic regression is usually performed by

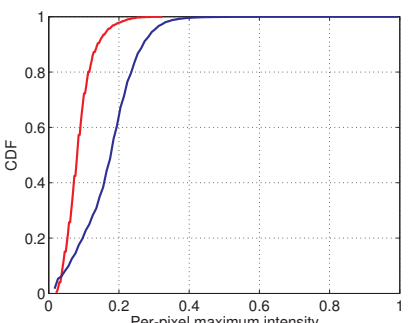

(a)

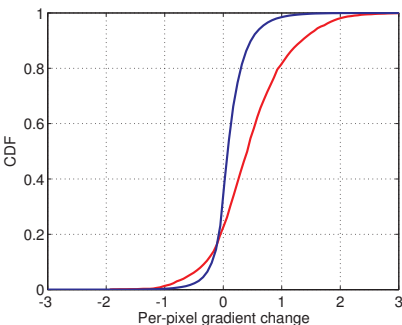

(c)

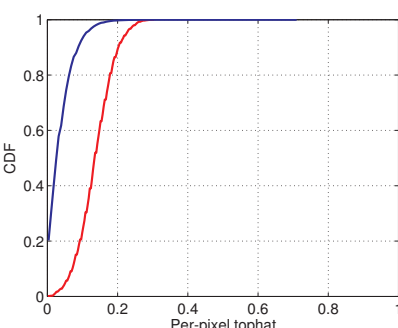

(b)

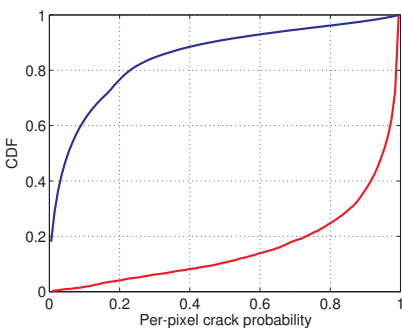

(d)
Fig. 5. $\quad \mathrm{CDFs}$ for the maximum light intensity $C_{1}$ (a), the close top-hat $C_{2}$ (b), the gradient change $S_{y y}$ (c), and the estimated crack probabilities (d) for manually identified cracks (red) and other parts of the slab sample (blue).

separating the classes at $50 \%$ class probability, but we select a higher, more conservative, required crack probability in order to limit the number of non-crack pixels in the resulting binary segmentation.

The segmentation phase is concluded by cleaning the thresholded binary image $T$ from clearly uninteresting parts. This is done by morphological filtering: False signatures at vertical edges are avoided by excluding pixels where a long vertical line can be fitted into the segmented set, using a morphological open top-hat. The exact length of the line should be long enough to avoid deleting cracks, and was here set to 25 pixels. Finally, small regions in the resulting binary image are excluded by an area opening, which avoids subsequent processing of a large number of very small regions (noise). In this work, regions containing less than 25 pixels were removed.

\section{Classification (P3)}

After segmentation potential cracks, i.e. segmented regions, are fed into another logistic regression classifier, but at this stage the classification is based on regional features (in contrast to the previous per-pixel classification). We denote identified regions as $R$ and their features $\mathbf{F}(R)$. A statistical classifier is defined based on the regional features orientation, width, solidity, and crack probability (as defined by (5)). These variables, extracted for each of the segmented regions, were selected from a larger set by lasso regularization, i.e. by introducing a bias towards zero for the coefficients [14]. A non-biased model was then created based on the resulting variables. Hence, the resulting model has an intended bias towards simplicity in terms of variables, but is without bias in the final coefficients. The width is calculated from a 


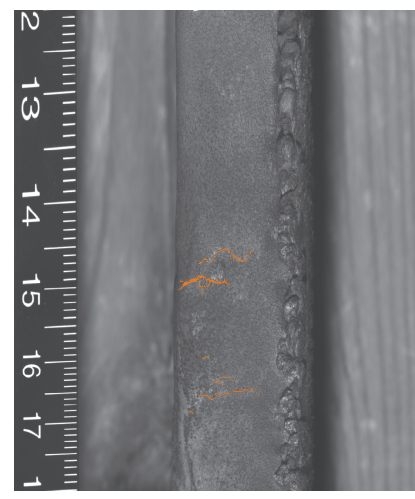

(a)

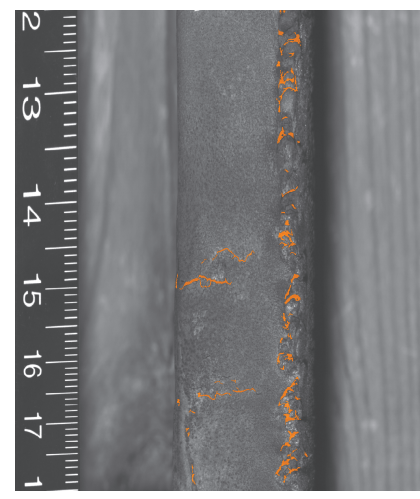

(b)

Fig. 6. Manual pixel classification (a) and the segmented regions, thresholded at $75 \%$ crack probability (b).

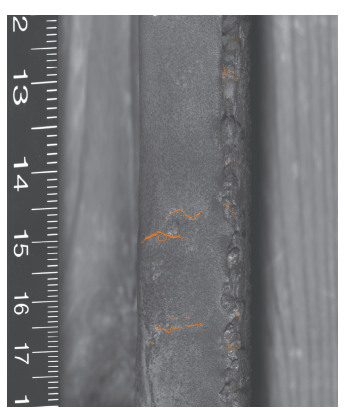

(a)

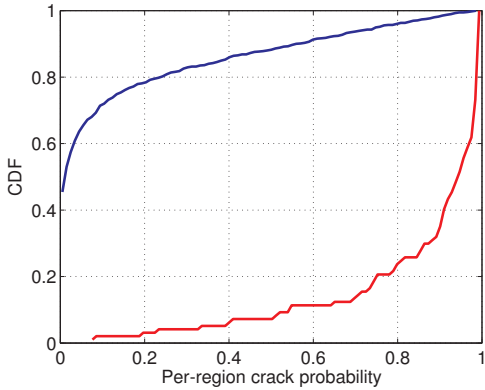

(b)
Fig. 7. Regional crack probabilities depicted in orange shading (a) and the total CDFs (b) for manually identified cracks (red) and other parts of the slab sample (blue).

pruned binary skeleton. Discarded variables included other geometrical properties such as length and area.

Instead of manually setting a strictly predefined classification threshold we set the regional crack probability $p(R \mid \beta, \mathbf{F})$ to the posterior probability for logistic regression, as in (5) but with another coefficient vector $\beta$ corresponding to the extracted regional features $\mathbf{F}(R)$. Choosing a threshold value then becomes a trade-off between high detection rate and a low number of false detections. It is therefore crucial that the resulting probability distributions for the two groups are well separated, which is indeed the case for the model set (see Fig. 7). For instance, a $70 \%$ threshold level for the regional crack probability keeps $86 \%$ of the segmented crack regions while discarding all but $6 \%$ of non-crack regions in the segmented set.

\section{RESULTS}

The described method was used to scan and process a validation set of images, resulting in a segmented set of potential crack regions with corresponding estimated crack probabilities. The validation set contained, just as the model set, in total over 50 corner crack samples of various sizes.

Figure. 8 shows an example of a captured front image from the validation set (Fig. 8a) and a few images for the same

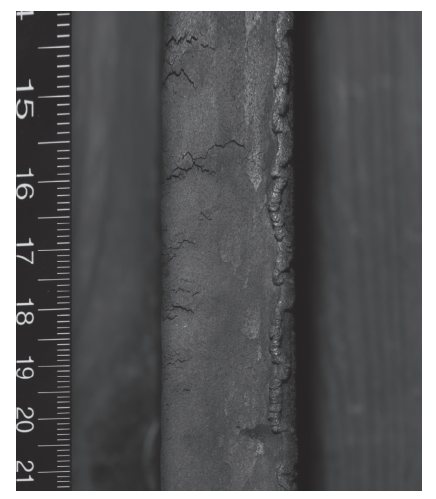

(a)

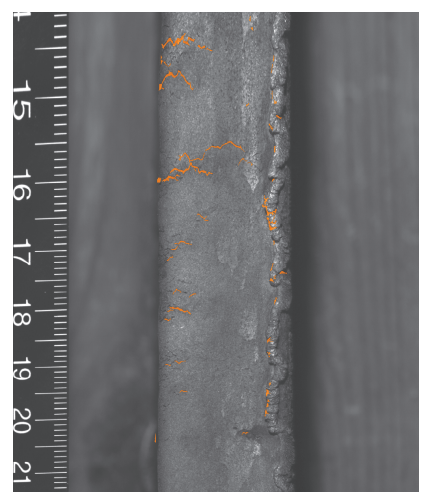

(c)

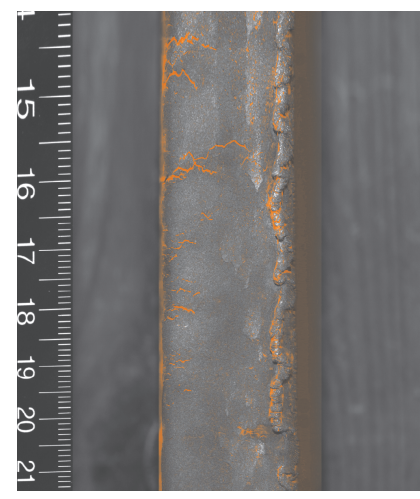

(b)

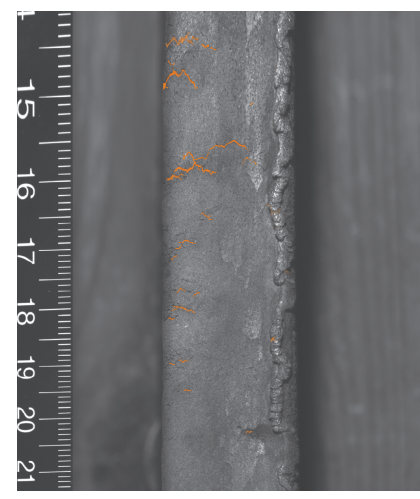

(d)
Fig. 8. An example from the validation set: The front image (a), the per-pixel posterior probabilities shaded in orange (b), the resulting binary segmentation (c), and the per-region crack probabilities for the segmented regions (d). The brightness of the orange shading is proportional to the assigned crack probability, i.e. regions with high crack probability are shaded in bright orange.

example during intermediate algorithm steps. The estimated per-pixel crack probabilities are displayed in Fig. 8b, where pixels with higher crack probability are shaded in brighter orange, and the segmentation resulting from thresholding this probability image at $75 \%$ is displayed in Fig. 8c. These regions are then fed to a classifier, providing per-region crack probabilities as displayed in Fig. 8d. The CDFs corresponding to Figs. $8 \mathrm{~b}$ and $8 \mathrm{~d}$ are shown in Fig. 9, and demonstrates good separation between true cracks and other regions. At the $75 \%$ per-pixel threshold, more than $70 \%$ of the manually identified pixels are correctly classified in the first step (while discarding 98\% of other pixels), while the second (regional) classification would at a $70 \%$ threshold level keep $78 \%$ of the segmented regions and discard all but 5\% of the remaining non-crack regions. Note that these threshold values can be easily varied depending on the preferences of the system user, allowing for trade-off between the number of successful detections and the risk for false detections. Furthermore, large scale usage would allow for collection of larger model sets and thereby more accurate probability estimations. 


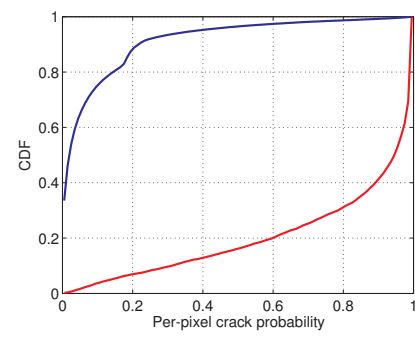

(a)

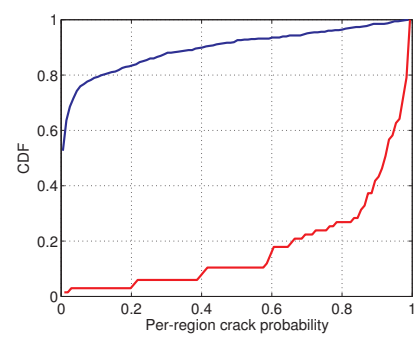

(b)
Fig. 9. CDFs for per-pixel crack probabilities (a) and per-region crack probabilities (b) for manually identified cracks (red) and other parts of the slab sample (blue).

\section{Discussion}

In order to measure the surface slope in the longitudinal direction, the two light sources are located one in front of and the other behind the point that is currently measured. The camera is of line-scan type, capturing transversal rows, which keeps the distances towards the light sources at constant length.

The $2^{\text {nd }}$ derivative yields signatures for cracks but is quite sensitive to small deviations in the data, resulting in a substantial amount of noise. It therefore needs to be combined with other features in order to achieve a robust crack detection system. We have here used the light intensity, since its robustness against artificial noise is a good complement to the more sensitive $2^{\text {nd }}$ derivative which is useful for finding the rapid slope changes that characterize the cracks. Using light intensity directly makes the system more dependent on the surface texture of the steel and the ambient light settings of the installation. Texture effects are handled by taking the morphological close top-hat into account, thereby considering not only the light intensity but also its relation to the surrounding region. The dependency on ambient light requires control of the surrounding environment in order to achieve robustness, but this can be quite simply addressed by shielding the measurement system from external light sources.

Looking at the results for manually classified data, the method indeed assigns high crack probabilities to crack regions and low crack probabilities to non-crack regions. We see that other transversal ditch-like features in the data are likely to be assigned a high crack probability, but this is a logical consequence of the algorithms - such regions do represent a change from negative to positive slope. By taking other parameters into account such false positives can be drastically reduced, which is demonstrated by the separation in region crack probability for the segmented data (as compared to the pixelwise crack probability used in the segmentation).

A key challenge in this type of application is the small size of defect regions, in relation to non-defect area. Since the cracks we are looking for make up such a low proportion of the steel surface, classification results must be very high in order to avoid false positives. The reason for this is that even at very low false positive classification rates the vast amount of non-defect data will still likely cause some non-crack regions being falsely identified as cracks. Hence, the possibility to find and use other additional extractable characteristics to improve results should be further pursued, with the goal of restricting the number of false positives to an absolute minimum.

Due to the adaptive structuring elements crack signatures can be emphasized using only one parameter. Using statistical methods to estimate crack probabilities also reduces the total number of required user-set parameters: instead of strictly defining for the data what we require, we leave much of this work to the data itself. The low number of parameters simplifies adjustments and analysis of the method. It should be noted that due to the data-driven segmentation and classification, a more representative model set should improve results substantially. In particular, one model set is most likely not suitable for all circumstances: for instance, different steel grades may have different properties. However, the fact that the segmentation is to a large extent depending on the model set makes it reasonably easy to create different models for different situations.

\section{CONClusion}

We have demonstrated a novel method for automated detection of small corner cracks in casted steel. Cracks in general cause high crack probability values, while only a minor proportion of other regions are assigned similar crack probabilities. More specifically: about $80 \%$ of the cracks present in the segmented regions are given a crack probability higher than $70 \%$, while the corresponding number for other non-crack regions is only $5 \%$. The segmented regions contain over $70 \%$ of the manually identified crack pixels. The results thereby provide proof-of-concept for the presented method.

\section{FUTURE WORK}

Due to the fact that the large amount of surface area not containing cracks is likely to cause false positives even with a very low rate of false classifications, future work should focus on decreasing the false positive classification rate even further. One option which can help to avoid false crack signatures from light intensity patterns originating from more largescale topological variations is to combine the photometric stereo images with 3D profile (range) data, which can assist substantially in defining the actual surface where we want to scan for cracks. More specifically, distinct surface height variations on the larger scale such as e.g. the slab border, scales, and scarfing borders can be more easily discarded from potential crack regions by using 3D data.

Higher resolution can be obtained not only by varying the speed of the camera in relation to the scanned surface, but also by varying the number of recorded scans required for each line in the image. This comes at the expense of higher noise levels due to fewer measurements being recorded for each point of the steel surface. Such effects on noise levels from increasing scanning speed should be investigated, in order to select an optimal measurement speed. 
Large scale testing and acquisition of data on-site is the natural next step, in order to provide good statistical evaluation of the method and thereby making a future prototype development possible. Comparison to other approaches also constitutes a topic for future work. Both of these are quite extensive tasks, however, and therefore lie beyond the scope of this work.

\section{ACKNOWLEDGMENT}

The authors would like to thank our measurement partners at Kemi-Tornio University of Applied Sciences (KTUAS), in particular Pauli Vaara who has been of great assistance in the work with the measurement setup as well as when performing the measurements.

This work was partly funded by the Swedish Steel Producers' Association, Jernkontoret.

\section{REFERENCES}

[1] B. Thomas, "On-line Detection of Quality Problems in Continuous Casting of Steel," in Proceedings of the International Symposium on Process Control and Optimization in Ferrous and Nonferrous Industry, TMS, Warrendale, PA,(Chicago, IL), 2003.

[2] P. Meilland, "Novel Multiplexed Eddy-Current Array for Surface Crack Detection on Rough Steel Surface," Proc. 9th ECNDT, Berlin, 2006.

[3] M. Allazadeh, C. Garcia, K. Alderson, and A. Deardo, "Ultrasonic image analysis of steel slabs," Advanced materials \& processes, vol. 166 , no. 12 , pp. 26-27, 2008.

[4] I. Alvarez, J. Marina, J. Enguita, C. Fraga, and R. Garcia, "Industrial online surface defects detection in continuous casting hot slabs," in Proceedings of SPIE, vol. 7389, 2009, p. 73891X.
[5] A. Landström and M. J. Thurley, "Morphology-based crack detection for steel slabs," IEEE Journal on Selected Topics in Signal Processing, vol. 6, no. 7, pp. 866-875, 2012.

[6] E. N. Malamas, E. G. Petrakis, M. Zervakis, L. Petit, and J.-D. Legat, "A survey on industrial vision systems, applications and tools," Image and Vision Computing, vol. 21, no. 2, pp. 171 - 188, 2003.

[7] R. Woodham, "Photometric method for determining surface orientation," Optical engineering, vol. 1, no. 7, pp. 139-144, 1980.

[8] R. Kozera, "Existence and uniqueness in photometric stereo," Applied Mathematics and Computation, vol. 44, no. 1, pp. 1-103, 1991.

[9] M. Drew, "Robust specularity detection from a single multi-illuminant color image," CVGIP: Image Understanding, vol. 59, no. 3, pp. 320327, 1994.

[10] G. Balschbach, J. Klinke, and B. Jhne, "Multichannel shape from shading techniques for moving specular surfaces," in Computer Vision ECCV98, ser. Lecture Notes in Computer Science, H. Burkhardt and B. Neumann, Eds. Springer Berlin Heidelberg, 1998, vol. 1407, pp. 170-184.

[11] D. Kang, Y. J. Jang, and S. Won, "Development of an inspection system for planar steel surface using multispectral photometric stereo," Optical Engineering, vol. 52, no. 3, pp. 039701-039701, 2013.

[12] S. K. Nayar and R. M. Bolle, "Reflectance based object recognition," International Journal of Computer Vision, vol. 17, no. 3, pp. 219-240, 1996.

[13] A. Landström and M. J. Thurley, "Adaptive morphology using tensorbased elliptical structuring elements," Pattern Recognition Letters, vol. 34, no. 12, pp. 1416-1422, 2013.

[14] R. Tibshirani, "Regression shrinkage and selection via the lasso," Journal of the Royal Statistical Society. Series B (Methodological), pp. 267-288, 1996 